(in press; The Year in Cognitive Neuroscience, Annals of the New York Academy of Sciences)

\title{
A vision of graded hemispheric specialization
}

\author{
Marlene Behrmann and David C. Plaut \\ Carnegie Mellon University, Pittsburgh, PA, USA
}

"Lateralization in the human brain: apparent or real?"

The Year in Cognitive Neuroscience 2015

Annals of the New York Academy of Sciences

Keywords: hemispheric specialization, lateralization, visual word form area, reading, word recognition, face recognition, fusiform face area, development, higher-order vision

Address for correspondence: Marlene Behrmann, Ph.D, Department of Psychology and the Center for the Neural Basis of Cognition, Carnegie Mellon University, Pittsburgh, PA, USA 15213-3890; email:

behrmann@cmu.edu

Acknowledgements: This research was supported by a grant from the National Science Foundation to MB and DP (BCS0923763) and by a grant to MB (\#SMA-1041755 to the Temporal Dynamics of Learning Center: PI: G. Cottrell). Thanks to Kevin Weiner, Ben Cipollini, Tina Liu, Eva Dundas, Suzy Scherf, Matt Lambon Ralph and Mark Vida for helpful discussion of some of the issues. 


\section{Abstract}

Understanding the process by which the cerebral hemispheres reach their mature functional organization remains challenging. We propose a theoretical account in which, in the domain of vision, faces and words come to be represented adjacent to retinotopic cortex by virtue of the need to discriminate among homogeneous exemplars. Orthographic representations are further constrained to be proximal to typically left-lateralized language-related information to minimize connectivity length between visual and language areas. As reading is acquired, orthography comes to rely more heavily (albeit not exclusively) on the left fusiform region to bridge between vision and language. Consequently, due to competition from emerging word representations, face representations that were initially bilateral become lateralized to the right fusiform region (albeit, again, not exclusively). We review recent research that describes constraints that

give rise to this graded hemispheric arrangement. We then summarize empirical evidence from a variety of studies (behavioral, evoked response potential, functional imaging) across different populations (children, adolescents and adults, left-handers and individuals with developmental dyslexia) that supports the claims that hemispheric lateralization is graded rather than binary and that this graded organization emerges dynamically over the course of development. Perturbations of this system either during development or in adulthood provide further insights into the principles governing hemispheric organization. 


\section{Introduction}

That which is inevitable does not have to be innate.

$$
\text { --- Jean Piaget }{ }^{1}
$$

The renowned developmental psychologist, Jean Piaget, argued that behaviors are not just determined by genetics or by the environment, but emerge from a complex interplay of both genetic and environmental factors in concert with biological, mathematical and physical constraints on the system. We propose that the same type of complex interplay applies to the emergence of functional organization of the brain and, in particular, to the way in which mature cerebral hemispheric lateralization manifests.

It is incontrovertible that, in the adult human, the two cerebral hemispheres differ both structurally and functionally, that each has a particular topographic organization, and that this organization is largely repeated across the majority of the population ${ }^{2,3}$. As Piaget reminds us, however, this robust and replicable outcome need not reflect a hard-wired, pre-specified architecture. Instead, the mature topographic organization of adult human cortex may result from a fundamental set of physiological constraints coupled with dynamic competitive and cooperative processes that play out in a consistent manner over the course of development and experience. In the current review, we provide empirical evidence to support this latter view, concluding that cerebral lateralization is real, rather than apparent, but that it is graded rather than categorical, and emerges from cortical dynamics rather then being architecturally pre-specified.

Hemispheric specialization is graded

Hemispheric asymmetries are abundant in the anatomy, neurochemistry and cytoarchitecture of the vertebrate brain. The crossed representation of the corticospinal motor system as well as the predominant crossing of sensory systems are well established [for recent review papers, see ${ }^{4}$ ]. 
The focus of the present review is whether, outside of primary sensory and motor areas of cortex--- and, in particular, outside of retinotopic visual areas--- functions are cleanly lateralized in the modal, right-handed adult individual. To address this issue, we explore two specific visual functions, whose neural basis lies beyond retinotopic cortex, and with which humans have abundant experience: the recognition of words and the recognition of faces. The received wisdom from decades of research, as reviewed below, is that the former is lateralized to the left hemisphere (LH) whereas the latter is lateralized to the right hemisphere $(\mathrm{RH})$. The evidence is gleaned from a variety of empirical sources and methodologies.

Neuropsychological studies have long indicated that a lesion to the LH occipitotemporal region in literate adults results in a reading deficit, termed pure alexia. The site of the lesion is typically in the medial occipitotemporal areas (with or without additional callosal damage), specifically along the fusiform and adjacent lingual gyri with possible incursion to the inferior longitudinal fasciculus ${ }^{5-8}$. In complementary fashion, a lesion to the $\mathrm{RH}$ occipitotemporal region results in an impairment in face recognition known as prosopagnosia ${ }^{9-12}$, and the site of the lesion in many, albeit not all, cases is to a region in the vicinity of the fusiform and lingual gyrus ${ }^{10,13}$. Of note, individuals with the congenital (also referred to as developmental) form of prosopagnosia have compromised integrity of the white matter tracts that project from occipital cortex to temporal (inferior longitudinal fasciculus; ILF) and frontal (inferior fronto-occipital fasciculus) cortex, and the extent to which the ILF is reduced in the RH is correlated with the severity of the prosopagnosia ${ }^{14}$ [also see ${ }^{15}$ ]. Similar findings of reduced white matter tracts have been observed in individuals with developmental dyslexia, as well, especially in the $\mathrm{LH}^{16,17}$ [also see $\left.{ }^{18}\right]$.

Compatible with this word-LH and face-RH distinction are extensive data from half-field or tachistoscopic studies showing dominance of the right visual field (LH) over the left visual field (RH) for visual word processing [for example ${ }^{19,20}$ ] and the reverse dominance for face processing [for review, see ${ }^{21}$ ]. Findings from many electrophysiological (ERP) studies also support this lateralization, with a stronger 
N170 component over the posterior $\mathrm{LH}$ than $\mathrm{RH}$ in response to visually presented words [for example, ${ }^{22,23}$ ] and a stronger $\mathrm{N} 170$ component over the $\mathrm{RH}$ than $\mathrm{LH}$ in response to visually presented faces [for example, $\left.{ }^{24,25}\right]$.

Taken together, there appears to be strong evidence for the segregation of word and face recognition to the $\mathrm{LH}$ and $\mathrm{RH}$, respectively. There are a number of straightforward explanations that might account for this segregation. Faces and words clearly differ in their overt geometry and image statistics, and computing representations for such diverse inputs in the same cortical area would not be a trivial endeavor. Additionally, faces and words diverge substantially in the nature and timing of their acquisition: face recognition develops relatively incidentally starting from a very early age whereas, for most individuals, word recognition is acquired through formal instruction schooling at around age 5 or 6 years. Finally, the evolutionary status of faces and words differ: face recognition has been a fundamental cognitive and social skill throughout the history of our species, whereas reading is a relatively recent invention, introduced approximately 5400 years ago ${ }^{26}{ }^{26}$ and, until roughly 150 years ago, was limited to a minority of the human population.

Notwithstanding the explanations offered for the segregation of face and word representations, close scrutiny of the majority of fMRI and ERP studies reveals a surprising degree of bilateral hemispheric activation for words and for faces; for examples, see ${ }^{27-32}$, albeit with relative weighting across the two hemispheres (see Figure 1): there is usually greater amplitude BOLD signal and significantly more voxels activated by words in the $\mathrm{LH}$ than $\mathrm{RH}\left[{ }^{29,33}\right.$; for review, see $\left.{ }^{34}\right]$ and the converse pattern holds true for faces. A similar pattern was observed in a recent study using high-density recording of surface field potentials in the ventral occipito-temporal cortex of patients with intractable epilepsy, revealing striking withinhemisphere interdigitation of face- and letter-string-selective zones ${ }^{35}$. Together, these findings undermine the argument for strict lateralization of face and word representations. The question then is what gives rise 
to the weighted asymmetry and, moreover, is the observed activation in the non-preferred hemisphere functionally relevant or simply epiphenomenal?

Insert Figure 1 approximately here

The origin of the weighted asymmetry across hemispheres

We have argued that the mature hemispheric organization, as reflected by the bilateral but weighted activation profiles for faces and words, arises over the course of development as a consequence of the operation of a set of general computational principles governing neural representation, processing and learning ${ }^{36,37}$. Even though word and face processing would seem to be completely unrelated visual domains, with different modes of acquisition, the theory provides a basis for understanding their interdependence.

At the core of the theory is the interplay between cooperation and competition among representations. The theory starts with the standard assumption that representations are hierarchically organized, such that the representation of information at each level cooperates with (i.e., mutually activates and strengthens) the representations of consistent information at lower and higher levels. Such cooperation depends on available connectivity, which strongly favors local connections in order to minimize axon length (and hence total white-matter volume) as well as to avoid errors as signal propagation distance increases or as inter-hemispheric engagement is necessary ${ }^{38}$. Short connection lengths lead to increased global efficiency and economy of network organization, are less burdensome metabolically and are more resilient to damage ${ }^{39}$. Such systems have been shown to be advantageous computationally too, revealing a number of desirable properties, including a tendency to decompose tasks into subtasks, to decouple the dynamics of recurrent subsystems, and to develop location-sensitive internal representations ${ }^{40}$. Within a given level, the representations of different (inconsistent) information compete 
with each other, both to become active and to become stronger through learning (e.g., by recruiting more neurons to encode them).

Despite their differences, both word and face recognition are both highly overlearned and place extensive demands on high-acuity vision given the high degree of visual similarity among exemplars. Thus, representations for both classes need to cooperate with (i.e., be connected to and, hence, be adjacent to) representations of central visual information; as a result, in both hemispheres, words and faces compete for representational space in areas adjacent to retinotopic cortex in which information from central vision is encoded $^{27,41-43}$ (see Figure 2). These areas end up becoming what are often referred to as the Visual Word Form Area (VWFA) in the LH, and the Fusiform Face Area (FFA) in the RH.

Insert Figure 2 here

To minimize connection length, orthographic representations are further constrained to be proximal to language-related information, which is left-lateralized in most individuals and, indeed, lateralization of word-selective activation is co-lateralized with language areas, as revealed in recent neuroimaging studies ${ }^{44,45}$. As a result, letters and words come to rely most heavily-albeit not exclusively - on the left fusiform region (VWFA) as an intermediate cortical region bridging between early vision and language. This idea is also consistent with the interactive view that left occipitotemporal regions become specialized for word processing because of top-down predictions from the language system integrating with bottom-up visual inputs ${ }^{34,46,47}$. As the LH region becomes increasingly tuned to represent words, the competition with face representations in that region increases. Consequently, face representations that were initially bilateral become more lateralized to the right fusiform region (FFA) albeit, again, not exclusively.

We have proposed that the pressure to minimize connection length between retinotopic visual cortex and LH language regions serves as the primary driving force behind the initiation of lateralization, but a host of other anatomical constraints may contribute as well, including the nature of cortical folds 
and other aspects of physical implementation of underlying neural hardware properties, such as cytoarchitectonics, receptor architectonics, myelination and white-matter connectivity ${ }^{48}$. Moreover, there is now increasing evidence that the cortical organization, as reflected in the cytoarchitectonic parcellation, is even more fine-grained than previously considered. For example, recent advancements in the delineation of the macroanatomical features of ventral visual cortex have revealed that the RH fusiform gyrus (FG) which mediates face representations is not a monolithic structure with a singular function; rather, the mid-fusiform gyrus indicates a cytoarchitectonic boundary which subdivides the FG into two areas, each with a different receptor architecture ${ }^{49}$ : FG1 serves as a transitional area between early and higher visual cortex whereas FG2 serves as a higher-order associative region ${ }^{48}$. $F G 2$ is also more strongly lateralized than FG1, and, in the LH, shows strong relations to the visual language processing system while, in the $\mathrm{RH}$, is more strongly associated with other face-selective regions. These findings indicate that functional lateralization of the FG may be dependent on differential connectivity patterns ${ }^{50,51}$. A similar distinction between two subregions of the left occipitotemporal VWFA region may also be observed ${ }^{48,52,}$ ${ }^{53}$; indeed, the nomenclature of VWFA-1 and VWFA-2 has been recently proposed ${ }^{54}$. The cytoarchitectonic and connectivity profiles of the two areas are currently undergoing further characterization (for similar findings regarding the impact of connectivity on functional properties of cortical regions, see ${ }^{15}$ ).

Similar ideas concerning connectivity and functional organization have been put forward previously; for example, according to Gazzaniga ${ }^{55}$, the corpus callosum in humans and the expansion of fast, myelinated fibers in particular regions of cortex both across and within species ${ }^{56,57}$ allow for the jettisoning of a preexisting system (for example, face recognition) as a new function develops in one hemisphere (for example, word recognition) while the other hemisphere can continue to perform the previous function for both hemispheres. Interestingly, Gazzaniga ${ }^{55}$ speculated that as the LH becomes more tuned for language competence, cortical tissue that had been dedicated to visuospatial processing may result in a decrement of those abilities in the LH. This subsequent 'deficit' in LH perceptual skills is evident in studies comparing the LH 
and $\mathrm{RH}$ in split-brain patients showing, for example, relative decrement in the $\mathrm{LH}$ versus $\mathrm{RH}$ ability to decide whether two objects are identical or mirror-reversed ${ }^{58,59}$. The consequences of language and reading for perceptual representation in the LH are also revealed in studies of individuals who are illiterate. Evidence from fMRI investigations has shown that illiterate individuals, relative to literate individuals, evince reduced LH activation to written strings and increased LH response to faces ${ }^{60,61}$. Of great interest, increasing literacy in such individuals leads to a shift in these hemispheric profiles with an increase in LH activation for words and an increase in $\mathrm{RH}$ lateralization for faces. Learning to read also leads to anatomical changes within the left intrahemispheric white matter pathways that interconnect these regions, specifically resulting in an increase in fractional anisotropy and a decrease in perpendicular diffusivity in the temporo-parietal portion of the left arcuate fasciculus ${ }^{62,63}$. Further evidence for this competitive account comes from studies showing decreases or pruning away of responses to non-preferred stimuli (e.g., shoes) in category-selective regions such as VWFA and FFA over the course of child development ${ }^{64}$.

If the critical trigger for the emergence of the left-lateralized VWFA is the pressure to couple visual and language areas, one might have predicted that the VWFA should be localized even more closely to the language regions in the anterior-posterior plane, or inferior-superior plane. There are, however, several reasons why the VWFA may not be more proximal to language regions. For example, there are properties of extrastriate visual cortex that are well suited to the processing of stimuli that appear near to the observer and require fine-grained discrimination (as would be true for words). In monkeys, face patches (but not color patches or scene patches) are modulated more strongly by near than by far stimuli ${ }^{65}$ and these near-disparity regions occupy foveal locations of the extrastriate eccentricity map permitting sufficient acuity ${ }^{65}$. Similar biases would be true for the recognition of words. Similarly, the locations of visual categories may depend on some kind of 'proto-organization', which is shape-based. This is well demonstrated in studies exploring the emergent category-selective organization in juvenile monkeys trained on a host of biologically relevant (e.g., faces) and cultural stimuli (e.g., text) 66,67 . The findings reveal that the acquisition of novel representations is constrained 
by some native organization and that this organization is superimposed on gradients of eccentricity (as noted above ${ }^{68}$ and curvature [see also ${ }^{69}$ ]. Taken together, there are several functional and anatomical constraints that likely codetermine the exact location of the VWFA in an individual, including a complex trade-off between proximity to bottom-up central and shape-based visual information, proximity to various types of languagerelated information (phonology, semantics), and constraints on organization of other visual recognition processes (that compete with words/faces).

\section{Predictions and further evidence}

The graded hemispheric theory makes a number of interesting and otherwise counterintuitive predictions, some of which have already been tested empirically. A key aspect of the theory concerns the notion that the LH lateralization for word recognition emerges over the course of development. Behavioral studies have previously documented an emerging right visual field/LH advantage for letters in a divided visual field task, as children develop the ability to name letters ${ }^{70,71}$. Consistent with this, cross-sectional developmental fMRI studies have also found a greater leftward asymmetry related to increases in age and linguistic skill as well as to the acquisition of word recognition ${ }^{72-74}$.

Relevant data from recent imaging studies also support the idea that the LH connectivity between visual and language areas is key to the emergence of hemispheric organization. One relevant study ${ }^{63}$ has revealed that the VWFA, compared with the fusiform face area, shows higher connectivity to lefthemispheric perisylvian superior temporal, anterior temporal and inferior frontal areas. Also of relevance is that the left occipitotemporal sulcus maps onto a peak of connectivity with language areas, with slightly distinct subregions showing preferential projections to areas respectively involved in grapheme-phoneme conversion and lexical access (see also ${ }^{75,76}$ ). Furthermore, that the reading-related ventral activations have the same topography in blind and sighted subjects suggests that connections with language may be critical and may serve as powerful constraints in determining the standard location of the VWFA ${ }^{77,78}$ even in the 
absence of visual input. Last, recent findings of greater interhemispheric effective connectivity between posterior cortex in children aged (mean) 4.5 years than in adults is also consistent with the hypothesis that there are changes in network properties that occur over development around the time that children are entering school and being exposed to orthography ${ }^{79}$.

The graded hemispheric specialization account makes the further specific predictions that, despite reading being evolutionarily much more recent than face recognition, word lateralization should precede face lateralization in development, and, moreover, the extent of reading acquisition should predict the degree of face lateralization across individuals. To test these predictions, we examined the hemispheric superiority for faces and words in children (7-9 years old), young adolescents (11-13 years old) and adults using a half-field discrimination task ${ }^{80}$. In this task (see Figure 3), a face or word was shown centered over fixation for $750 \mathrm{~ms}$ (so that the youngest children could encode the stimulus) and, immediately thereafter, a stimulus was presented for $150 \mathrm{msec}$ (too brief a time to program a saccade) in either the right or left visual field. The participant was required to decide whether or not the second stimulus was the same as the first by pressing one of two keys to indicate same/different (responses counterbalanced across participants) ${ }^{80}$. Whereas all three age groups showed the expected right visual field (LH) advantage for words, only the adults showed a left visual field $(\mathrm{RH})$ advantage for faces (Figure 4a). Moreover, as predicted, the emergence of face lateralization was correlated with reading competence, measured on an independent standardized test, after regressing out age, quantitative reasoning scores and face discrimination accuracy (see Figure 4b).

Insert Figure 3 approximately here Insert Figure $4 \mathrm{a}$ and $4 \mathrm{~b}$ approximately here

An EEG study used the identical experiment to compare the ERP responses for a new group of children (aged 7-12 years) versus adults to ascertain whether the lateralization of orthographic 
representations precedes the lateralization of face representations. First, the behavioral data collected while the ERP signal was being measured replicated the above result ${ }^{81}$ : adults showed the complementary right and left visual field superiority for words and faces, respectively, while children showed only the right visual field superiority for words. Additionally, whereas for the adults there was a larger N170 ERP component in the $\mathrm{LH}$ over $\mathrm{RH}$ for words, and conversely, a larger N170 in the RH over LH for faces, the children revealed only the adult hemispheric pattern for words, but no ERP hemispheric superiority for faces (see Figure 5). Of particular interest, the magnitude of the children's N170 for faces in the RH was correlated with that of the N170 for words in their LH. These findings suggest that the neural mechanisms for face and word recognition do not develop independently, and that earlier LH word lateralization drives later RH face lateralization. This is a particularly counterintuitive finding given that one's experience with face recognition precedes the exposure to orthography by many years. To be clear, the claim is not that young children are unable to recognize faces at all (although it is well established that their performance is far from adult level ${ }^{82}$ ); rather, the idea is that face lateralization may be bilaterally organized initially and only becomes more right-lateralized when pressured by the more left-lateralized acquisition of word representations (see also ${ }^{64,83}$ ).

Insert Figure 5 approximately here

A further prediction of the account is that any failure to develop coherent word representations in the LH should affect not only word lateralization but should also adversely impact face lateralization. Indeed, upholding this prediction, left-handed individuals who, as a group, show more variable language dominance do not show obvious lateralization for face processing, as measured by the strength of the N170 ERP component ${ }^{84}$ or by fMRI investigation ${ }^{85}$. Relatedly, right-handed adults with a diagnosis of developmental dyslexia and ongoing reading difficulties, are both impaired at face perception, relative to matched controls ${ }^{86}$, and, intriguingly, do not show the typical profile of LH-word and RH-face superiorities 
and organization ${ }^{87,88}$. Finally, literacy impacts the manner in which faces are processed - thus, with increasing literacy, there is a decrease in automatic holistic processing in the $\mathrm{LH}$, perhaps enabling the use of a more analytic and flexible processing strategy ${ }^{89}$ [for related results in altered tuning of the visual system in illiterates acquiring orthographic representations, see ${ }^{90}$ ].

The claim that it is the lateralization of language that constrains the emergence of word (and then face) lateralization is also well supported by findings showing that the left superior temporal activation for spoken language correlates with the lateralization of occipitotemporal activations for both written words and faces ${ }^{91}$ and that the degree of face-selectivity in the RH varies with handedness ${ }^{92}$. Also consistent with the precedence of language and the subsequent cascade of lateralization is a study that compared face and word activation fMRI profiles in monozygotic and dizygotic twins. The results of this study indicate that activations of the left VWFA and the temporal speech areas are partially under genetic control whereas activation of the face-selective $\mathrm{RH}$ regions is primarily influenced by individual experience ${ }^{91}$. One interpretation of these findings is that LH word selectivity is directly dependent on genetically-specified connectivity with other LH language areas, whereas $\mathrm{RH}$ face selectivity is the consequence of protracted reading experience. In the same study, the $\mathrm{RH}$ occipital face area (OFA) also appeared to be at least partially influenced by genetics, but because the LH posterior face selective region was not considered, the degree to which there are hemispheric differences in the relative contributions of genetic and experiencedependent factors to the development of the OFA remains unclear.

Finally, data from developmental studies confirm that the LH superiority for orthographic over nonorthographic stimuli is not present in pre-literate children and becomes apparent only after reading instruction and practice ${ }^{93,94}$. This LH lateralization increases with reading ability ${ }^{95,96}$ and the N170 continues to strengthen through adolescence ${ }^{73,97}$. This experience-dependent neural signature can also be observed in adulthood as the increased selectivity for learned orthography is seen both functionally in adults learning to read a second language $e^{98}$, and structurally in adults learning to read a first language ${ }^{47}$. 
Taken together, the findings reviewed thus far are consistent in supporting a model of bilateral but graded representations of faces and words.

\section{Functional relevance of the activation in the non-preferred hemisphere}

The data thus far are consistent in supporting a model of bilateral but graded representations of faces and words. A critical question that remains to be addressed, though, is whether the activation in the nonpreferred hemisphere is merely epiphenomenal or is functionally relevant. Evidence supporting the latter view comes predominantly from the study of individuals who were premorbidly normal in face and word recognition but who subsequently suffered a unilateral cortical lesion in the vicinity of the left or right ventral occipitotemporal cortex. One recent study addressed this by quantifying the speed and accuracy of word and face recognition in four pure alexic and three prosopagnosic individuals (and matched controls), all of whom suffered a unilateral cortical lesion. The results showed that the pure alexic (LH damage) patients demonstrated mild but reliable face recognition deficits and that the prosopagnosic (RH damage) patients revealed mild but reliable word recognition deficits. Similarly, in a group of 32 patients with unilateral right or left lesions, a significant number of patients with LH damage were impaired at face perception and a significant number of patients with $\mathrm{RH}$ lesions were impaired at word recognition, leading to the conclusion that face recognition and word reading may be mediated by distributed neural systems that are more bilaterally organized than commonly assumed ${ }^{99}$. Also consistent with these findings are the results from a study of eight patients with pure alexia who, relative to controls, were slower and less accurate at face naming and name-face matching ${ }^{41}$.

The contribution of the non-preferred hemisphere is also inferred from the finding that prosopagnosia is more severe following bilateral than unilateral right lesions ${ }^{13}$, implicating a LH contribution to face recognition, and that prosopagnosia has been reported in a right-hander subsequent to a LH lesion ${ }^{100,101}$. In complementary fashion, pure alexia has been reported in a right-hander after a 
unilateral right occipitotemporal lesion ${ }^{102,103}$ and, in one patient with pure alexia, the recovered reading was subsequently disrupted by transcranial magnetic stimulation to the $\mathrm{RH}$ but not to the $\mathrm{LH}^{104}$.

Together, these neuropsychological investigations suggest that both hemispheres participate in both face and word recognition. Whether the contribution of the two hemispheres is not only differentially weighted but qualitatively different is rather less clear. Some of the patient studies cited above report that signature characteristics of pure alexia (for example, the word length effect) are also observed in the prosopagnosic individuals (albeit to a lesser extent) but that the signature characteristics of prosopagnosia (relatively better performance on inverted than upright faces) are not observed in the pure alexic patients ${ }^{105}$. One recent study that investigated the face perception skills of pure alexic individuals in some detail observed that the sequential part-based processing strategy that gives rise to the length effect in the reading of these patients apparently allows them to discriminate between faces on the basis of feature identity. In these individuals, the processing of second-order configural information is compromised due to their left pFG lesion, suggesting that the deficit in face perception that emerges after a LH lesion is a reflection of the damage to the LH part-processing bias per se rather than being a direct parallel of prosopagnosia after a RH lesion ${ }^{106}$.

Some $\mathrm{fMRI}$ studies have also uncovered differences in activation profile across the two hemispheres: for example, the $\mathrm{RH}$ is more activated when matching whole faces than when matching face parts, whereas this pattern of activity was reversed in the left homologous region ${ }^{107}$. A related study showed that the $\mathrm{RH}$ activation is more activated by categorical face/non-face judgments whereas the $\mathrm{LH}$ activation was more correlated with image-level face resemblance ${ }^{108}$. On the other hand, using MVPA and recursive feature elimination, Nestor and colleagues showed that the right and left fusiform gyri have very similar sensitivity estimates for face identity discrimination ${ }^{109}$. Clearly, additional research is needed to determine the extent and nature of the differences in the bilateral hemispheric representations of faces and of words. 
What really gives rise to the word-left and face-right distinction?

We have suggested that the trigger for the emergence of hemispheric specialization comes from the combination of LH language (as is the case for most right-handed individuals), demands for high-acuity vision and constraints to keep connection length short. One might even question why language is unilaterally organized and why language areas arise in the LH in the first place. One possibility is that the need for fine and rapid motor control for speech (and perhaps demanding manual tasks) requires that a single neural system drives motor production because bilateral systems would need perfect synchronisation to drive motor output efficiently (and, in fact, some theories of stuttering suggest that it reflects bilateral control speech).

However, the question remains about why there is LH dominance for language in the majority of the population rather than $\mathrm{RH}$ dominance or perhaps random assignment across the hemispheres. The answer to this is rather more speculative: perhaps the LH instantiates a bias that is specific to language, such as the ability to detect rapid transitions from one stimulus to another ${ }^{110}$, tuning to roughly syllable-length temporal rhythms, or some other type of genetically pre-determined bias (for review, see ${ }^{111,112}$ ). Numerous hypotheses have been proposed to bridge the genetics of hemispheric dominance and handedness, and while we do not review these exhaustively, a few are of particular relevance. For instance, a generic trait of asymmetry such as handedness, which may be genetically determined [for example, see ${ }^{113,114}$ ], and which also correlates with the lateralization of language ${ }^{115-117}$, may have a broad impact on several aspects of functional hemispheric specialization. These different possibilities (e.g., fundamental computational properties of LH, genetics) are not mutually exclusive. A recent paper using Near-infrared Spectroscopy (NIRS) with infants concludes that lateralization for language emerges out of the interaction between preexisting left-right biases in generic auditory processing (signal-driven hypothesis), and a left-hemisphere predominance of particular learning mechanisms (learning-biases hypothesis) ${ }^{118}$. 
A host of other fundamental encoding asymmetries may also contribute to lateralization and potentially to the LH language bias. These include, for example, a LH bias to processing parts of stimuli rather than wholes ${ }^{107}$ or a bias to encode inputs in a more categorical (LH) rather than coordinate (RH) fashion ${ }^{119}$. The bias may also arise from greater LH efficiency in processing high spatial frequencies in the visual input ${ }^{43}$, although recent evidence suggests that this may, in fact, be a consequence rather than a cause of the hemispheric lateralization ${ }^{120}$. Structural differences such as differences in connectivity ${ }^{121}$ or minicolumnar organization ${ }^{122,123}$, among others, may also contribute to lateralization and some structural asymmetries seem to be present from birth and are sex-related ${ }^{124}$ [although note that a large study of cortical surface area did not reveal any difference between left and right handers in any cortical region ${ }^{125}$ ].

Lastly, hemispheric differences may not be immutable, even in adulthood, and may be substantially modulated by task demands. For example, in an imaging study exploring attentional differences, in both hemispheres, larger population receptive fields were associated with attending to a visual stimulus compared with attending to a central fixation point ${ }^{126}$. Also, in the $\mathrm{LH}$ but not $\mathrm{RH}$, attending to the stimulus also resulted in more peripheral preferred locations of contralateral representations, compared with attending fixation. Similarly, the left visual field advantage for chimeric faces with emotional expression is thought to be the result of the strategic use of $\mathrm{RH}$ processing mechanisms ${ }^{127}$.

Together, these findings raise more questions than provide clear answers. The question of the origin of the LH language bias continues to perplex investigators and, as evident from above, many hypotheses have been offered but no clear and definitive conclusion has been reached. A deeper understanding of the nature of hemispheric dominance, its underlying basis and its relationship to handedness, too, is clearly needed but is beyond the scope of this paper.

Implications for recovery

A key principle of the graded hemispheric view that we have articulated is that both word 
and face representations are bilaterally implemented, albeit with weighted asymmetry, and that this mature pattern is the result of competition for representational space in the LH during the process of reading acquisition. There may well be additional advantages that accrue from graded hemispheric specialization both because this arrangement makes the system more robust to damage and provide some degree of capacity for post-damage plasticity-related recovery ${ }^{128}$. If a normal functional system is primarily based on two bilateral-interconnected neural regions, then damage to one or other side will, in the most simplistic terms, represent a reduction in the total available computational resources. The total amount of damage not only changes the severity of the initial impairment but also will limit the amount of plasticityrelated recovery, and this will probably interact with any graded specializations arising from differential connectivity.

If both face and word representations are indeed bilateral, one might ask why, after a unilateral lesion, a deficit in word recognition (pure alexia) or in face recognition (prosopagnosia) persist. Why doesn't the intact hemisphere come to mediate recognition, especially if, as we have claimed, the representations in the non-preferred hemisphere play a functional role and are not merely epiphenomenal? The existing evidence, however, suggests otherwise and, to date, there is rather little reported recovery of function in pure alexia ${ }^{129}$ or in acquired forms of prosopagnosia ${ }^{130} 131$.

One possible explanation for this apparent contradiction concerns individual differences in the extent of word-LH and face-RH lateralization even among right-handed individuals. According to the graded hemisphere account, there may be different degrees of competition and cooperation between the hemispheres across individuals, resulting in different profiles of asymmetric weighting across individuals. These differential competitive/cooperative dynamics may be the consequence of a host of factors. One possible factor is the size of the corpus callosum as a larger corpus callosum size is associated with less lateralization. This is true for different subregions of the corpus callosum, as well as for the corpus callosum as a whole. For example, the midbody of the callosum, which carries fibers to and from motor cortex, is 
larger in individuals who have less lateralization in handedness ${ }^{132,133}$. Also, the callosal cross-sectional area is proportionally larger for left-handers ${ }^{134}$, who, as a group, show less functional cortical lateralization than right-handers. Within humans, a larger corpus callosum size is also associated with a larger number of thin fibers (less myelinated, less conductive) compared with thick fibers ${ }^{56}$ and indeed, axons of the posterior superior temporal lobe, which are associated with language, are asymmetrically myelinated ${ }^{135}$. Moreover, development of lateralization interacts with the development of the corpus callosum, and callosal fibers, which are among the longest connections in the brain ${ }^{136}$, are among the most variable and unreliable fibers during development.

One might speculate then, that across the population, the extent of lateralization for face and word recognition might vary such that, at one end of the distribution, there might be a cohort of individuals who are strongly lateralized for both face and word perception (see Figure 6). In such individuals, the competition for hemispheric representation may play out most strongly over the course of development, resulting in a strong imbalance of representation. At the other end of the continuum, however, there might be a cohort with much more balanced hemispheric representation, perhaps even approximating bilateral representation of faces and words in some cases. At a population level, this would give rise to the account we have espoused above, but individual differences might also exist within the population. One possibility, then, is that those few individuals who exhibit pure alexia or prosopagnosia after a lesion might be those who, premorbidly, had the greatest degree of lateralization of word and face representation. If so, then perhaps the minimal representations in the non-preferred hemisphere do not suffice to support recovery of normal performance in these more strongly lateralized individuals. It may not be surprising then that both pure alexia and prosopagnosia are rather rare and that recovery and recovery is minimal or difficult in such cases. This notion suggests that one might be able to take a large sample of individuals with posterior damage (for example, following posterior cerebral artery infarction) and determine how many individuals with lesions to the vicinity of the LH VWFA and the RH FFA go on to 
become pure alexic or prosopagnosic. The hypothesis put forward here is that only a small subset of individuals would have these disorders, namely just those individuals with extreme premorbid lateralization.

An alternative possibility is that, even in less lateralized individuals, the opportunities for compensation from the structurally intact, non-preferred hemisphere are hampered by inhibitory effects from the lesioned hemisphere. Konen and colleagues ${ }^{137}$ showed that, although the structurally intact LH of an individual with visual agnosia following damage to the right lateral occipital complex (LOC) was activated in response to visual stimuli, its response profile was markedly disrupted with the result that effects such as object-selectivity and adaptation were not observed even in the intact hemisphere. There is growing consideration of the impact of interhemispheric dynamics and there is evidence to suggest that, for example, noninvasive neuromodulation of the contralesional hemisphere may trigger adaptive neuroplastic changes in individuals with severe chronic stroke $e^{138}$, further attesting to the possibility of transcallosal inhibition or excitation.

\section{Extension of principles to functional organization of other cortical regions}

We have proposed an account in which functional organization results from some minimal physiological constraints and biases, and that competition and cooperation shapes the topography of ventral visual cortex. Similar arguments have been made in the context of lateralization of other cognitive skills, as well. One such example concerns the lateralization of function of the superior temporal cortex (STS) in humans. Whereas the left superior temporal cortex is specialized for language processes, the right superior temporal cortex has become dominant for a different function, namely serving as a multimodal matrix for the exploration of objectand space-related information in the surroundings ${ }^{139,140}$. Recent evidence attest to the complementary relationship of language and spatial attention; in individuals with $\mathrm{RH}$ language specialization, the $\mathrm{LH}$ is dominant for spatial attention and the converse holds for individuals with LH language dominance ${ }^{141}$. It is argued, 
however, that the lateralized segregation of function of STS has occurred during evolution, as the pre-existing LH bias for language emerges over phylogenetic, rather than ontogenetic, change.

The claim of bilateral representation of function in the anterior temporal lobe (ATL) with only graded specialization reflecting differential connectivity, has also been proposed ${ }^{142,143}$. A large-scale meta-analysis of the functional neuroimaging literature on left versus right ATL function reports strong bilaterality of ATL activations except for naming/speech production and the processing of written words - both of which are proposed to follow from differential connectivity to left lateralised speech production and (graded) orthographic-recognition areas $^{144}$.

\section{Summary and conclusions}

One of the most striking findings in the study of high-level vision over the last twenty years is the discovery of cortical areas that are selective for particular behaviorally relevant categories, like face recognition or word recognition. Not only are these cortical areas found in most individuals (humans and non-human primates), but each area has a stereotypical anatomical location and connectivity pattern, as well. These regions are typically interpreted as implementing specialized processing for particular categories that exist due to behavioral or even evolutionary pressures. Mounting evidence suggests, however, that this specialization need not be predetermined and that, rather, the mature profile of hemispheric specialization may arise from integrative processes and dynamic interactions across multiple distributed regions and systems. Our account claims that there are some constraints which bias the functional organization of ventral cortex and these include structural/maturational factors such as eccentricity and curvature biases and bias for short connection length. Critically, the confluence of these minimal biases may suffice for the eventual mature hemispheric organization profile and there is no need to stipulate the locations and functions of a LH VWFA and a RH FFA a priori. In essence, some minimal assumptions about pre-existing structural or functional constraints can result in impressive functional organization of ventral cortex. 
We have proposed an account of graded hemispheric specialization based on competitive interactions between face and word representation for foveally-biased cortex, constrained by the need to integrate reading with a largely left-lateralized language system ${ }^{36,37}$. The computational approach explains how the organization of a complex system can emerge from the interaction of a number of basic physiological constraints ${ }^{40,145}$.

This perspective on functional brain organization is likely to be a fruitful avenue for future research and, although our account is focused on the hemispheric organization of face and word representations, a similar competitive account has been espoused for the hemispheric organization of language vis-à-vis spatial attention ${ }^{146}$ and of the organization of the anterior temporal lobe ${ }^{144}$. Finally, individual differences in connectivity profile, and cooperative and competitive functions can result not only in different profiles of lateralization but may also result in individual differences in differences in inter-hemispheric integration ${ }^{147}$, intelligence $^{148,149}$, and performance in different cognitive domains ${ }^{150}$ (for further review, see ${ }^{151}$ ). 
Figure legends

Figure 1: Average activation map showing preferential activation to letter strings (purple) and faces (yellowred). The maps are presented on a Talairach-normalized inflated brain (center). The same data are superimposed on flattened right and left hemispheres. Note the bilateral activation of both faces and letter strings with asymmetry across the two hemispheres. OT, occipito-temporal sulcus; pFs, posterior fusiform gyrus; CoS, collateral sulcus. (From Hasson et al., 2002).

Figure 2: (A) Character-Related Activation and Retinotopic Borders Preferential activation to letter strings (purple), and faces (yellow-red) shown on the inflated and unfolded brain of one representative subject (JM). Red dotted lines denote borders of retinotopic visual areas V1, V2, V3, VP, V3A, and V4/V8. Note that the posterior letters-related region encroaches on the foveal representation of early retinotopic areas, while the occipitotemporal region is within the anterior lateraloccipital complex. (From Hasson et al., 2002).

Figure 3: Schematic depiction of experiment to assess hemispheric advantage for face and word representations. For both stimulus types, a fixation screen appears first followed by a central stimulus, which is encoded. Thereafter, a brief fixation appears and is immediately followed by a stimulus presented briefly to the left or right visual field. The participant makes a same/different decision between the central and the lateral stimulus. (From Dundas et al., 2013).

Figure 4: a. Mean accuracy and +/-1 SE for (a) adults, (b) young adolescents, and (c) children as a function of stimulus type (faces/words) for the left and right visual field presentation. b. Scatterplot and correlation analysis showing a significant relationship between reading comprehension percentile (on the standardized 
Education Records Bureau examination) and degree of face lateralization (LVF-RVF) for the young adolescents and children. LVF= left visual field; RVF =right visual field. (From Dundas et al., 2013).

Figure 5: (a) Adult group ERP waveforms for words and faces in the LH and the RH. (b) Child group ERP waveforms for words and faces in the $\mathrm{LH}$ and the $\mathrm{RH}$. (c) The topographic scalp distributions of the mean amplitude between 160 and $220 \mathrm{msec}$ for words and faces in both groups. Note that blue (negative) is more lateralized to the left for words and right for faces in the adults but not in the children. (From Dundas et al., 2014).

Figure 6: Individual differences. Functional activation profiles from four different individuals, two of whom show bilateral activation for faces and for words and two of whom show only unilateral activation for faces $(\mathrm{RH})$ and for words (LH). 


\section{References}

1. Piaget, J., Structuralism. 1970, New York: Basic Books.

2. Amunts, K., et al., BigBrain: an ultrahigh-resolution 3D human brain model. Science, 2013. 340(6139): p. 1472-5.

3. Brancucci, A., Electroencephalographic and magnetoencephalographic indices of hemispheric asymmetry, in The two halves of the brain, K. Hugdahl and R. Westerhausen, Editors. 2010, MIT Press: Cambridge, MA. p. 211-250.

4. Hugdahl, K. and K. Westerhausen, The two halves of the brain, ed. (Eds.). 2010, Cambridge, MA: MIT Press.

5. Salvan, C.V., et al., Visual object agnosia and pure word alexia: correlation of functional magnetic resonance imaging and lesion localization. J Comput Assist Tomogr, 2004. 28(1): p. 63-7.

6. Feinberg, T.E., et al., Associative visual agnosia and alexia without prosopagnosia. Cortex, 1994. 30: p. 395-412.

7. Cohen, L., et al., The pathophysiology of letter-by-letter reading. Neuropsychologia, 2004. 42(13): p. 1768-1780.

8. Cohen, L., et al., Visual word recognition in the left and right hemispheres: Anatomical and functional correlates of peripheral alexias. Cerebral Cortex, 2003. 13: p. 1313-1333.

9. Gainotti, G. and C. Marra, Differential contribution of right and left temporo-occipital and anterior temporal lesions to face recognition disorders. Front Hum Neurosci, 2011. 5: p. 55.

10. Bouvier, S.E. and S.A. Engel, Behavioral deficits and cortical damage loci in cerebral achromatopsia. Cereb Cortex, 2006. 16(2): p. 183-91.

11. Damasio, A., H. Damasio, and D. Tranel, Prosopagnosia: Anatomic and physiological aspects, in Aspects of face processing, H.D. Ellis, et al., Editors. 1986, Martinus Nijhoff: Dordrecht. p. 279-290.

12. Barton, J.J.S., Disorder of higher visual function. Curr Opin Neurol, 2011. 24(1): p. 1-5.

13. Barton, J.J.S., Structure and function in acquired prosopagnosia: lessons from a series of 10 patients with brain damage. J Neuropsychol, 2008. 2(Pt 1): p. 197-225.

14. Thomas, C., et al., Reduced structural connectivity in ventral visual cortex in congenital prosopagnosia. Nat Neurosci, 2009. 12(1): p. 29-31.

15. Gomez, J., et al., Functionally defined white matter reveals segregated pathways in human ventral temporal cortex associated with category-specific processing. Neuron, 2015. 85(1): p. 216-27.

16. Vandermosten, M., et al., A qualitative and quantitative review of diffusion tensor imaging studies in reading and dyslexia. Neurosci Biobehav Rev, 2012. 36(6): p. 1532-52.

17. Klingberg, T., et al., Miscrostructure of temporo-parietal white matter as a basis for reading ability: Evidence from diffusion tensor magnetic resonance imaging. Neuron, 2000. 25: p. 493-500.

18. Wandell, B.A., The neurobiological basis of seeing words. Ann N Y Acad Sci, 2011. 1224: p. 63-80.

19. Hellige, J.B., B. Laeng, and C. Michimata, Processing asymmetries in the visual system, in he two halves of the brain: Information processing in the cerebral hemispheres R. Hugdahl and K. Westerhausen, Editors. 2010, MIT Press: Cambridge, MA. p. 379-415.

20. Levy, J., et al., Are variations among right-handed individuals in perceptual asymmetries caused by characteristic arousal differences between hemispheres? J Exp Psychol Hum Percept Perform, 1983. 9(3): p. 329-59.

21. Young, A.W., Functions of the right cerebral hemisphere. 1983, London, UK: Academic Press.

22. Maurer, U., B. Rossion, and B.D. McCandliss, Category specificity in early perception: face and word $n 170$ responses differ in both lateralization and habituation properties. Front Hum Neurosci, 2008. 2: p. 18.

23. Mercure, E., et al., Differential lateralization for words and faces: category or psychophysics? J Cogn Neurosci, 2008. 20(11): p. 2070-87. 
24. Scott, L.S. and C.A. Nelson, Featural and configural face processing in adults and infants: A behavioral and electrophysiological investigation. Perception, 2006. 35: p. 1107-1128.

25. Scott, L.S., et al., A reevaluation of the electrophysiological correlates of expert object processing. J Cogn Neurosci, 2006. 18(9): p. 1453-65.

26. Dehaene, S. and L. Cohen, Cultural recycling of cortical maps. Neuron, 2007. 56(2): p. 384-98.

27. Hasson, U., et al., Center-biased representation for characters in the human ventral visual stream. Neuron, 2002. 34: p. 479-490.

28. Kanwisher, N., J. McDermott, and M.M. Chun, The fusiform face area: a module in human extrastriate cortex specialized for face perception. J Neurosci, 1997. 17(11): p. 4302-11.

29. Puce, A., et al., Differential sensitivity of human visual cortex to faces, letterstrings, and textures: $A$ functional magnetic resonance imaging study. The Journal of Neuroscience, 1996. 16(16): p. 52055215.

30. Sergent, J. and J.L. Signoret, Functional and anatomical decomposition of face processing: evidence from prosopagnosia and PET study of normal subjects. Philos Trans R Soc Lond B Biol Sci, 1992. 335(1273): p. 55-61; discussion 61-2.

31. Price, C.J. and A. Mechelli, Reading and reading disturbance. Curr Opin Neurobiol, 2005. 15(2): p. 2318.

32. Tagamets, M.A., et al., A parametric approach to orthographic processing in the brain: An fMRI study. Journal of Cognitive Neuroscience, 2000. 12(2): p. 281-297.

33. Cohen, L., et al., The visual word form area: spatial and temporal characterization of an initial stage of reading in normal subjects and posterior split-brain patients. Brain, 2000. 123 ( Pt 2): p. 291-307.

34. Price, C.J. and J.T. Devlin, The interactive account of ventral occipitotemporal contributions to reading. Trends Cogn Sci, 2011. 15(6): p. 246-53.

35. Matsuo, T., et al., Alternating Zones Selective to Faces and Written Words in the Human Ventral Occipitotemporal Cortex. Cerebral Cortex, 2015. 25: p. 1265-1277.

36. Plaut, D.C. and M. Behrmann, Complementary neural representations for faces and words: A computational exploration. Cogn Neuropsychol, 2011. 28(3-4): p. 251-75.

37. Behrmann, M. and D.C. Plaut, Distributed circuits, not circumscribed centers, mediate visual recognition. TRENDS in Cognitive Sciences, 2013. 17(5): p. 210-9.

38. Cherniak, C., Component placement optimization in the brain. J Neurosci, 1994. 14(4): p. 2418-27.

39. Vertes, P.E. and E.T. Bullmore, Annual research review: Growth connectomics--the organization and reorganization of brain networks during normal and abnormal development. J Child Psychol Psychiatry, 2015. 56(3): p. 299-320.

40. Jacobs, R.A. and M.I. Jordan, Computational Consequences of a Bias toward Short Connections. Journal of Cognitive Neuroscience, 1992. 4(4): p. 323-336.

41. Roberts, D.J., et al., Efficient visual object and word recognition relies on high spatial frequency coding in the left posterior fusiform gyrus: evidence from a case-series of patients with ventral occipitotemporal cortex damage. Cereb Cortex, 2013. 23(11): p. 2568-80.

42. Levy, l., et al., Center-periphery organization of human object areas. Nat Neurosci, 2001. 4(5): p. 533-9.

43. Woodhead, Z.V., et al., Dissociation of sensitivity to spatial frequency in word and face preferential areas of the fusiform gyrus. Cereb Cortex, 2011. 21(10): p. 2307-12.

44. Cai, Q., et al., The left ventral occipito-temporal response to words depends on language lateralization but not on visual familiarity. Cereb Cortex, 2010. 20(5): p. 1153-63.

45. Van der Haegen, L., Q. Cai, and M. Brysbaert, Colateralization of Broca's area and the visual word form area in left-handers: fMRI evidence. Brain Lang, 2012. 122(3): p. 171-8.

46. Devlin, J.T., et al., The role of the posterior fusiform gyrus in reading. J Cogn Neurosci, 2006. 18(6): p. 911-22.

47. Carreiras, M., et al., An anatomical signature for literacy. Nature, 2009. 461(7266): p. 983-6. 
48. Grill-Spector, K. and K.S. Weiner, The functional architecture of the ventral temporal cortex and its role in categorization. Nat Rev Neurosci, 2014. 15(8): p. 536-48.

49. Caspers, J., et al., Receptor architecture of visual areas in the face and word-form recognition region of the posterior fusiform gyrus. Brain Struct Funct, 2015. 220(1): p. 205-19.

50. Caspers, J., et al., Functional characterization and differential coactivation patterns of two cytoarchitectonic visual areas on the human posterior fusiform gyrus. Hum Brain Mapp, 2014. 35(6): p. 2754-67.

51. Weiner, K.S., et al., The mid-fusiform sulcus: a landmark identifying both cytoarchitectonic and functional divisions of human ventral temporal cortex. Neuroimage, 2014. 84: p. 453-65.

52. Ben-Shachar, M., R.F. Dougherty, and B.A. Wandell, White matter pathways in reading. Curr Opin Neurobiol, 2007. 17(2): p. 258-70.

53. Ben-Shachar, M., et al., Differential sensitivity to words and shapes in ventral occipito-temporal cortex. Cereb Cortex, 2007. 17(7): p. 1604-11.

54. Stigliani, A., K.S. Weiner, and K. Grill-Spector, Temporal processing capacity in high-level visual cortex is domain-specific. in revision, 2015.

55. Gazzaniga, M., Cerebral specialization and interhemispheric communication. Brain, 2000. 123: p. 12931326.

56. Aboitiz, F. and J. Montiel, One hundred million years of interhemispheric communication: the history of the corpus callosum. Braz J Med Biol Res, 2003. 36(4): p. 409-20.

57. Olivares, R., J. Montiel, and F. Aboitiz, Species differences and similarities in the fine structure of the mammalian corpus callosum. Brain Behav Evol, 2001. 57(2): p. 98-105.

58. Corballis, P.M., et al., Illusory contour perception and amodal boundary completion: Evidence of a dissociation following callosotomy. Journal of Cognitive Neuroscience, 1999. 11(4): p. 459-466.

59. Funnell, M.G., P.M. Corballis, and M.S. Gazzaniga, A deficit in perceptual matching in the left hemisphere of a callosotomy patient. Neuropsychologia, 1999. 37(10): p. 1143-54.

60. Dehaene, S., et al., How learning to read changes the cortical networks for vision and language. Science, 2010. 330(6009): p. 1359-64.

61. Dehaene, S., et al., Illiterate to literate: behavioral and cerebral chamges induced by reading acquisition. Nature Reviews Neuroscience, 2015. 16: p. 234-244.

62. Thiebaut de Schotten, M., et al., Learning to read improves the structure of the arcuate fasciculus. Cereb. Cortex 2012 24(4): p. 989-995.

63. Bouhali, F., et al., Anatomical connections of the visual word form area. J Neurosci, 2014. 34(46): $p$. 15402-14.

64. Cantlon, J.F., et al., Cortical representations of symbols, objects, and faces are pruned back during early childhood. Cereb Cortex, 2011. 21(1): p. 191-9.

65. Verhoef, B.E., K.S. Bohon, and B.R. Conway, Functional architecture for disparity in macaque inferior temporal cortex and its relationship to the architecture for faces, color, scenes, and visual field. J Neurosci, 2015. 35(17): p. 6952-68.

66. Srihasam, K., J.L. Vincent, and M.S. Livingstone, Novel domain formation reveals proto-architecture in inferotemporal cortex. Nat Neurosci, 2014.

67. Srihasam, K., et al., Behavioral and anatomical consequences of early versus late symbol training in macaques. . Neuron, 2012. 73: p. 608-619.

68. Hasson, U., et al., Large-scale mirror-symmetry organization of human occipito-temporal object areas. Neuron, 2003. 37(6): p. 1027-41.

69. Op de Beeck, H.P., et al., A stable topography of selectivity for unfamiliar shape classes in monkey inferior temporal cortex. Cereb Cortex, 2008. 18(7): p. 1676-94.

70. Jablonowska, K. and W. Budhoska, Hemispheric differences in the visual analysis of the verbal and nonverbal material in children. . Acta Neurobiol. Exp, 1976. 36: p. 693-701. 
71. Davidoff, J.B. and D.J. Done, A longitudinal study of the development of visual field advantage for letter matching. Neuropsychologia, 1984. 22(3): p. 311-8.

72. Schlaggar, B.L., et al., Functional neuroanatomical differences between adults and school-age children in the processing of single words. Science, 2002. 296(5572): p. 1476-9.

73. Schlaggar, B.L. and B.D. McCandliss, Development of neural systems for reading. Annu Rev Neurosci, 2007. 30: p. 475-503.

74. Turkeltaub, P.E., et al., Development of neural mechanisms for reading. Nat Neurosci, 2003. 6(7): p. 767-73.

75. Yeatman, J.D., A.M. Rauschecker, and B.A. Wandell, Anatomy of the visual word form area: Adjacent cortical circuits and long-range white matter connections. Brain and Language, 2013. 125: p. 146-155.

76. Wandell, B.A., A.M. Rauschecker, and J.D. Yeatman, Learning to see words. Annu. Rev. Psychol. , 2012. 63: p. 31-53.

77. Reich, L., et al., A ventral visual stream reading center independent of visual experience. Curr Biol, 2011. 21(5): p. 363-8.

78. Striem-Amit, E., et al., Reading with sounds: sensory substitution selectively activates the visual word form area in the blind. Neuron, 2012. 76(3): p. 640-52.

79. He, W., et al., Development of effective connectivity in the core network for face perception. Hum Brain Mapp, 2015.

80. Dundas, E.M., D.C. Plaut, and M. Behrmann, The joint development of hemispheric lateralization for words and faces. Journal of experimental psychology. General, 2013. 142(2): p. 348-58.

81. Dundas, E.M., D.C. Plaut, and M. Behrmann, An ERP investigation of the co-development of hemispheric lateralization of face and word recognition. Neuropsychologia, 2014. 61C: p. 315-323.

82. Carey, S. and R. Diamond, Are faces perceived as configurations more by adults than by children? Visual Cognition, 1994. 1(2/3): p. 253-274.

83. Li, S., et al., Neural competition as a developmental process: early hemispheric specialization for word processing delays specialization for face processing. Neuropsychologia, 2013. 51(5): p. 950-9.

84. Dundas, E.M., D.C. Plaut, and M. Behrmann, Variable Left-hemisphere Language and Orthographic Lateralization Reduces Right-hemisphere Face Lateralization. J Cogn Neurosci, 2014: p. 1-13.

85. Bukowski, H., et al., Cerebral lateralization of face-sensitive areas in left-handers: only the FFA does not get it right. Cortex, 2013. 49(9): p. 2583-9.

86. Gabay, Y., et al., Atypical perceptual processing of faces in developmental dyslexia. Neuropsychologia, in press.

87. Dundas, E., et al., Electrophysiological profile of word and face recognition in adults with developmental dyslexia. submitted manuscript.

88. Monzalvo, K., et al., Cortical networks for vision and language in dyslexic and normal children of variable

socio-economic status. Neuroimage, 2012. 61: p. 258-274.

89. Ventura, P., et al., Literacy acquisition reduces the influence of automatic holistic processing of faces and houses. Neurosci Lett, 2013. 554: p. 105-9.

90. Dunabeitia, J.A., K. Orihuela, and M. Carreiras, Orthographic Coding in Illiterates and Literates. Psychol Sci, 2014. 25(6): p. 1275-1280.

91. Pinel, P., et al., Genetic and Environmental Influences on the Visual Word Form and Fusiform Face Areas. Cereb Cortex, 2014.

92. Willems, R.M., M.V. Peelen, and P. Hagoort, Cerebral lateralization of face-selective and body-selective visual areas depends on handedness. Cereb Cortex, 2010. 20(7): p. 1719-25.

93. Maurer, U., D. Brandeis, and B.D. McCandliss, Fast, visual specialization for reading in English revealed by the topography of the N170 ERP response. Behav Brain Funct, 2005. 1: p. 13. 
94. Maurer, U., et al., Emerging neurophysiological specialization for letter strings. J Cogn Neurosci, 2005. 17(10): p. 1532-52.

95. Marcel, T., L. Katz, and M. Smith, Laterality and reading proficiency. Neuropsychologia, 1974. 12(1): $p$. 131-9.

96. Shaywitz, B.A., et al., Disruption of posterior brain systems for reading in children with developmental dyslexia. Biol Psychiatry, 2002. 52(2): p. 101-10.

97. Posner, M.I. and B.D. McCandliss, Brain circuitry during reading, in Converging methods for understanding reading and dyslexia, R.M. Klein and P.A. McMullen, Editors. 1999, MIT Press: Cmabridge, MA. p. 305-338.

98. Baker, C.I., et al., Visual word processing and experiential origins of functional selectivity in human extrastriate cortex. Proc Natl Acad Sci U S A, 2007. 104(21): p. 9087-9092.

99. Gerlach, C., et al., No strong evidence for lateralization of word reading and face recognition deficits following posterior brain injury. Journal of Cognitive Psychology, 2014. http://dx.doi.org/10.1080/20445911.2014.928713.

100. Mattson, A.J., H.S. Levin, and J. Grafman, A case of prosopagnosia following moderate closed head injury with left

hemisphere focal lesion. Cortex, 2000. 36(1): p. 125-137.

101. Anaki, D., et al., Associative (prosop)agnosia without (apparent) perceptual deficits: a case-study. Neuropsychologia, 2007. 45(8): p. 1658-71.

102. Davous, P. and F. Boller, Transcortical alexia with agraphia following a right temporo-occipital hematoma in a right-handed patient. Neuropsychologia, 1994. 32(10): p. 1263-72.

103. Ogden, J.A., Dyslexia in a right-handed patient with a posterior lesion of the right cerebral hemisphere. Neuropsychologia, 1984. 22(3): p. 265-80.

104. Coslett, H.B. and N. Monsul, Reading with the right hemisphere: Evidence from transcranial magnetic stimulation. Brain and Language, 1994. 46: p. 198-211.

105. Behrmann, M. and D.C. Plaut, Bilateral hemispheric processing of words and faces: evidence from word impairments in prosopagnosia and face impairments in pure alexia. Cereb Cortex, 2014. 24(4): p. 1102-18.

106. Roberts, D.J., et al., Processing deficits for familiar and novel faces in patients with left posterior fusiform lesions. Cortex, 2015. in press.

107. Rossion, B., et al., Hemispheric asymmetries for whole-based and part-based face processing in the human fusiform gyrus. J Cogn Neurosci, 2000. 12(5): p. 793-802.

108. Meng, M., et al., Lateralization of face processing in the human brain. Proc Biol Sci, 2012. 279(1735): p. 2052-61.

109. Nestor, A., D.C. Plaut, and M. Behrmann, Unraveling the distributed neural code of facial identity through spatiotemporal pattern analysis. Proc Natl Acad Sci U S A, 2011. 108(24): p. 9998-10003.

110. Tallal, P., S. Miller, and R.H. Fitch, Neurobiological basis of speech: a case for the preeminence of temporal processing. Ann N Y Acad Sci, 1993. 682: p. 27-47.

111. MacNeilage, P., The origin of speech. 2008, New York: Oxford University Press USA.

112. Corballis, M.C., Lateralization of the human brain. Prog Brain Res, 2012. 195: p. 103-21.

113. McManus, I.C., The inheritance of left-handedness. Ciba Found Symp, 1991. 162: p. 251-67; discussion 267-81.

114. Ocklenburg, S., et al., The ontogenesis of language lateralization and its relation to handedness. Neurosci Biobehav Rev, 2014. 43: p. 191-8.

115. Knecht, S., et al., Handedness and hemispheric language dominance in healthy humans. Brain, 2000. 123 Pt 12: p. 2512-8.

116. Knecht, S., et al., Language lateralization in healthy right-handers. Brain, 2000. 123 ( Pt 1): p. 74-81. 
117. Szaflarski, J.P., et al., Language lateralization in left-handed and ambidextrous people: fMRI data. Neurology, 2002. 59(2): p. 238-44.

118. Minagawa-Kawai, Y., A. Cristià, and E. Dupoux, Cerebral lateralization and early speech acquisition: $A$ developmental scenario. . Developmental Cognitive Neuroscience, 2011. 1: p. 217-232.

119. Kosslyn, S.M., et al., Evidence for two types of spatial representations: Hemispheric specialization for categorical and coordinate relations. Journal of Experimental Psychology: Human Perception and Performance, 1989. 15(4): p. 723-735.

120. Ossowski, A. and M. Behrmann, Left hemisphere specialization for word reading is not contingent on a left lateralized bias for high spatial frequency visual information. Cortex, 2015. in press.

121. Hsiao, J.H., B. Cipollini, and G.W. Cottrell, Hemispheric asymmetry in perception: a differential encoding account. J Cogn Neurosci, 2013. 25(7): p. 998-1007.

122. Chance, S.A., The cortical microstructural basis of lateralized cognition: a review. Front Psychol, 2014. 5: p. 820.

123. Hutsler, J. and R.A. Galuske, Hemispheric asymmetries in cerebral cortical networks. Trends Neurosci, 2003. 26(8): p. 429-35.

124. Li, G., et al., Mapping longitudinal hemispheric structural asymmetries of the human cerebral cortex from birth to 2 years of age. Cereb Cortex, 2014. 24(5): p. 1289-300.

125. Guadalupe, T., et al., Differences in cerebral cortical anatomy of left- and right-handers. Front Psychol, 2014. 5: p. 261.

126. Sheremata, S.L. and M.A. Silver, Hemisphere-dependent attentional modulation of human parietal visual field representations. J Neurosci, 2015. 35(2): p. 508-17.

127. Coronel, J.C. and K.D. Federmeier, Task demands modulate decision and eye movement responses in the chimeric face test: examining the right hemisphere processing account. Front Psychol, 2014. 5: p. 229.

128. Schapiro, A.C., et al., Why Bilateral Damage Is Worse than Unilateral Damage to the Brain. Journal of Cognitive Neuroscience, 2013. 25(12): p. 2107-2123.

129. Starrfelt, R., R.R. Olafsdottir, and I.M. Arendt, Rehabilitation of pure alexia: a review. Neuropsychol Rehabil, 2013. 23(5): p. 755-79.

130. DeGutis, J.M., et al., Face processing improvements in prosopagnosia: successes and failures over the last 50 years. Front Hum Neurosci, 2014. 8: p. 561.

131. Woodhead, Z.V., et al., Reading therapy strengthens top-down connectivity in patients with pure alexia. Brain, 2013. 136(Pt 8): p. 2579-91.

132. Witelson, S.F., Hand and sex differences in the isthmus and genu of the human corpus callosum. A postmortem morphological study. Brain, 1989. 112 ( Pt 3): p. 799-835.

133. Luders, E., et al., When more is less: associations between corpus callosum size and handedness lateralization. Neuroimage, 2010. 52(1): p. 43-9.

134. Witelson, S.F., The brain connection: the corpus callosum is larger in left-handers. Science, 1985. 229(4714): p. 665-8.

135. Anderson, B., B.D. Southern, and R.E. Powers, Anatomic asymmetries of the posterior superior temporal lobes: a postmortem study. Neuropsychiatry Neuropsychol Behav Neurol, 1999. 12(4): p. 247-54.

136. Lewis, J.D., et al., The relation between connection length and degree of connectivity in young adults: a DTI analysis. Cereb Cortex, 2009. 19(3): p. 554-62.

137. Konen, C.S., et al., The functional neuroanatomy of object agnosia: a case study. Neuron, 2011. 71(1): p. 49-60.

138. Demirtas-Tatlidede, A., et al., Long-term effects of contralesional rTMS in severe stroke: Safety, cortical excitability, and relationship with transcallosal motor fibers. NeuroRehabilitation, 2015. 36(1): p. 51-9. 
139. Karnath, H.-O., S. Ferber, and M. Himmelbach, Spatial awareness is a function of the temporal not the posterior parietal lobe. Nature, 2001. 411: p. 950-953.

140. Karnath, H.O., New insights into the functions of the superior temporal cortex. 2001. 2: p. 568-576.

141. Cai, Q., L. Van der Haegen, and M. Brysbaert, Complementary hemispheric specialization for language production and visuospatial attention. Proc Natl Acad Sci U S A, 2013. 110(4): p. E322-30.

142. Lambon Ralph, M.A., Neurocognitive insights on conceptual knowledge and its breakdown. Philos Trans R Soc Lond B Biol Sci, 2014. 369(1634): p. 20120392.

143. Lambon Ralph, M.A., et al., No right to speak? The relationship between object naming and semantic impairment: neuropsychological evidence and a computational model. J Cogn Neurosci, 2001. 13(3): p. 341-56.

144. Rice, G.E., M.A. Lambon Ralph, and P. Hoffman, The Roles of Left Versus Right Anterior Temporal Lobes in Conceptual Knowledge: An ALE Meta-analysis of 97 Functional Neuroimaging Studies Cerebral Cortex, 2014. doi:10.1093/cercor/bhv024

145. Jacobs, R.A., Nature, nurture and the development of functional specializations: A computational approach. Psychonomic Bulletin and Review, 1997. 4(3): p. 299-309.

146. Suchan, J. and H.O. Karnath, Spatial orienting by left hemisphere language areas: a relict from the past? Brain, 2011. 134(Pt 10): p. 3059-70.

147. Shimono, M., H. Mano, and K. Niki, The brain structural hub of interhemispheric information integration for visual motion perception. Cereb Cortex, 2012. 22(2): p. 337-44.

148. Li, Y., et al., Brain anatomical network and intelligence. PLoS Comput Biol, 2009. 5(5): p. e1000395.

149. Bassett, D.S., et al., Cognitive fitness of cost-efficient brain functional networks. Proc Natl Acad Sci U S A, 2009. 106(28): p. 11747-52.

150. Cole, M.W., et al., Global connectivity of prefrontal cortex predicts cognitive control and intelligence. J Neurosci, 2012. 32(26): p. 8988-99.

151. van den Heuvel, M.P. and O. Sporns, Network hubs in the human brain. Trends Cogn Sci, 2013. 17(12): p. 683-96. 